\title{
Surgical Management of Gut-Tie in a Cow
}

\author{
Harmanpreet Singh Sodhi*, Balreet Singh Sidhu and Ashwani Kumar
}

Department of Veterinary Surgery and Radiology, College of Veterinary Science, Guru Angad
Dev Veterinary and Animal Sciences University, Ludhiana-141004, Punjab, India

*Corresponding author

\section{A B S T R A C T}

Gut-tie is a rare clinical entity and it often presents a diagnostic challenge which may result in life-threatening consequences. This case report describes diagnosis and successful surgical management of gut-tie in a crossbred cow. A 3.5 year old HF crossbred cow weighing $412 \mathrm{~kg}$

\section{Keywords}

Bovine, Intestinal surgery, Strangulating obstruction

\section{Article Info}

Accepted:

07 July 2019

Available Online:

10 August 2019 was presented with a history of anorexia and impaction from 7 days. There was history of pain and loss of defecation from 4 days. Animal was 8 month pregnant. Cow had tachycardia $(120 / \mathrm{min})$ and tachypnea (28/min) with rumen hypermotility (4/2 min). Per rectal examination detected dilated intestinal loops and mucoid secretions in the rectum. Ultrasonographic examination found dilated intestines $(4.9 \mathrm{~cm})$ and scanty effusions in the reticular region. Marked neutrophilic $(96 \%)$ leukocytosis $(18600 / \mu \mathrm{L})$ was detected with toxic neutrophils and left shift. A tentative diagnosis of complete small intestinal obstruction was made. Right flank exploratory laparotomy done under local anesthesia (2\% lignocaine hcl) revealed marked distention of the small intestines. On further exploration, a loop of the small intestine was found incarcerated within the tight fibrous band, which was extending from the caudal right abdominal wall toward the pelvic inlet. The band was visualized by bringing it out of incision. This band was resected to make the incarcerated intestines free. Postoperative medication included broad spectrum antibiotics, analgesics, fluid therapy and supportive medication. Animal passed feces within 6 hours of surgery and recovered uneventfully. This case report puts on record the diagnosis and successful surgical management of a rare case of gut-tie in a crossbred cow.

\section{Introduction}

Gut-tie or pelvic hernia refers to an obstruction of intestines that is thought to cause from recoil of the spermatic cord following castration by traction in growing cattle (Scott et al., 1997; Lores et al., 2006). The cattle with obstructive intestinal disease often present a diagnostic challenge and may result in immediate life-threatening consequences. Several disease conditions may lead to obstruction in the flow of ingesta through the intestinal tract such as internal herniation through the rents in the mesentery or greater omentum (Pardon et al., 2009) or involving the round ligament of the liver (Robertson, 1979; Coetzee, 2000), between fibrotic bands (Pearson, 1973) or through congenital openings (Koller et al., 2001) or intestinal strangulation by persistent urachal remnants (Mesaric and Modic, 2003). Signs of obstructive intestinal disease include a reduction in the volume of faeces or failure to pass feces, abdominal distension with areas of 
tympanitic resonance on the right side of the abdomen and colic. Differentiation of the causes of abdominal pain and intestinal obstruction is usually posing a challenge to the bovine practitioner / surgeon and exploratory surgery is necessary for arriving a definitive diagnosis and treatment. This case report puts on record the diagnosis and successful surgical management of a rare case of gut-tie due to fibrous band in a crossbred cow.

\section{History and Clinical Signs}

A 3.5 year old, $412 \mathrm{~kg} \mathrm{HF}$ cross was presented to the Large Animal Clinic at the Guru Angad Dev Veterinary and Animal Sciences University, Ludhiana, India with a history of impaction from 7days. Animal was anorectic since then. There was a history of pain from 4days and animal was not a passing faeces onlymucus was coming out from rectum.Water intake was also reduced but animal was urinating normally. There was also a history of bloat from yesterday. Animal was 8 month pregnant. The animal was healthy prior to the onset of this illness.

Previous treatment given by local veterinarian was liquid paraffin, bloatosil, rumentas without any improvement in the condition. Otherwise the cow was active from its general appearance. Vitals of animal at the time of presentation were normal rectal temperature $\left(101.2^{0} \mathrm{~F}\right)$, tachycardia (heart rate $120 /$ minute) and tachypnea (respiration rate 28 /minute), pink mucus membrane and hyper motility of rumen ( $4 / 2$ minutes).

Per rectal examination revealed total loss of faeces from rectum. Rectum was empty. One tense fold of mesentery was palpated in mid abdomen. Intestines were felt dilated along with around 8 month pregnant uterus. On complete blood examination, hemoglobin (12.5 gram\%), total erythrocyte count $\left(7.02 \times 10^{6} \%\right.$ cubic millimeter), packed cell volume $(37.6 \%)$ and platelet $\left(419 \times 10^{3} /\right.$ cubic millimeter) were normal. Marked neutrophilic (neutrophils 96\%) leukocytosis (total leucocyte count was 18600/cubic millimeter) and corresponding lymphopenia (lymphocytes 4\%) giving an interpretation of neutrophilic leukocytosis with many toxic neutrophils and left shift.

Percutaneous ultrasonographic examination was carried out on standing animal in cattle crate using $5 \mathrm{MHz}$ convex transducer as described earlier (Singh et al., 2017). It revealed no abscess or fluid in the reticular region. Only mild reaction is seen in reticular region. Intestines were markedly dilated upto $4.9 \mathrm{~cm}$ with reduced motility (Fig. 1). Duodenum was also dilated with marked ileus (Fig. 2). Pylorus was dilated and fluid filled (Fig. 3). Abomasum appeared dilated and caecum could not be seen. Fetus and amniotic fluid was seen. Upon radiography of reticular area, no foreign body was seen and diaphragmatic line was clear. A tentative diagnosis of complete small intestinal strangulation and obstruction was made and the cow was prepared for a right-flank exploratory laparotomy.

\section{Treatments and Discussion}

Right flank exploratory surgery was performed with the cow standing in a crate. The right paralumbar fossa was prepared by clipping hair. The right paralumbar fossa was anesthetized by proximal paravertebral block at T13-L1, L1-L2, and L2-L3 using 2\% lidocaine hydrochloride. After aseptic preparation of the surgical site, a $20 \mathrm{~cm}$ vertical incision was made over skin in the mid right paralumbar fossa. The muscular layers were incised and omentum was retracted cranially to open peritoneal cavity. Exploration of the peritoneal cavity revealed marked distention of the small intestine. The caecum was collapsed and position/size of the 
abomasum was normal. A systematic exploration of the peritoneal cavity revealed that a part of small intestine (jejunum) was trapped within tight fibrous bands (Fig. 4), which extended from the caudal abdominal wall toward the pelvic inlet, leading to incarceration. The band was visualized by bringing it out of incision. The incarcerated small intestine was assessed to be healthy, normal colored and viable. Resection of the band was performed to relieve intestinal obstruction. The caudal intestines were checked for patency and the surgical wound was closed in routine fashion. Cow passed feces within 6 hours of surgery and recovered uneventfully. Histopathology of the band like structure causing intestinal obstruction, confirmed to be a fibrous band (Fig. 5 and 6).

Postoperative medication included broad spectrum antibiotics (inj. Ampicillin + Cloxacillin @ $10 \mathrm{mg} / \mathrm{kg}$, twice a day, IM for 5 days), analgesic (inj. Meloxicam @ 0.2 $\mathrm{mg} / \mathrm{kg}$, once daily, IM for 3 days), fluid therapy (inj. Normal saline solution 10 liter, IV daily for 3 days) and supportive medication ( 2 boli of yeast and $50 \mathrm{~g}$ of pulvLiv 52, once daily orally for 7 days) along with daily antiseptic dressing using povidone iodine. Surgical wound healed uneventfully and skin sutures were removed after 14 days.

The clinical signs observed in the current study were non-specific for intestinal ileus suggestive of intestinal obstruction that may be a result of intraluminal (Kumar et al., 2015; Singh et al., 2017) or extraluminal causes (Pearson, 1973; Coetzee, 2000; Koller et al., 2001; Mesaric and Modic, 2003; Pardon et al., 2009). Leukocytosis maybe associated with mild endotoxaemia or early peritonitis in cases on intestinal obstruction and ileus (Anderson et al., 1993) or it may be stress induced in sick cattle.

Fig.1 Ultrasonogram at right flank showing dilated intestinal loops

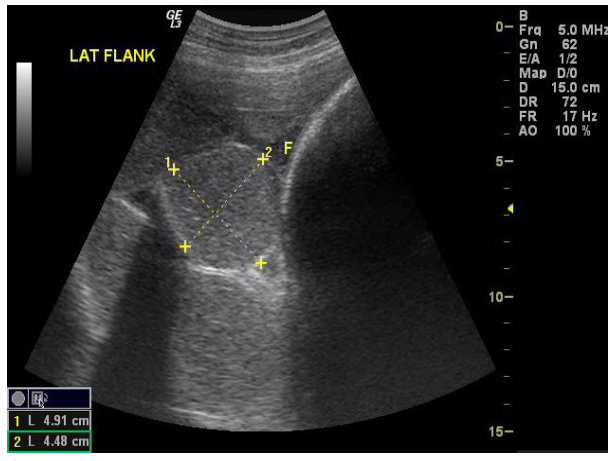

Fig.2 Ultrasonogram from right $11^{\text {th }}$ ICS showing dilated duodenum

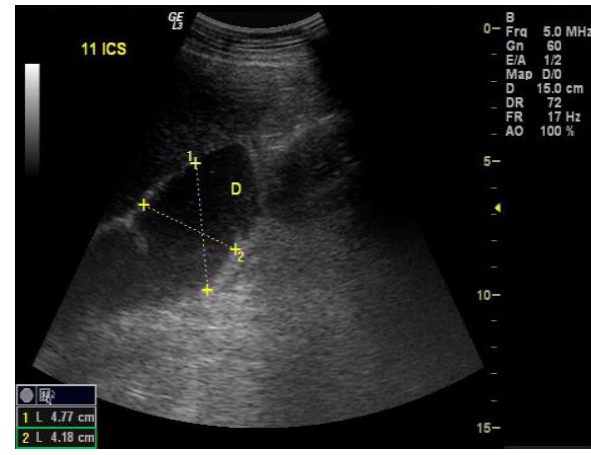


Fig.3 Ultrasonogram showing dilated pylorus

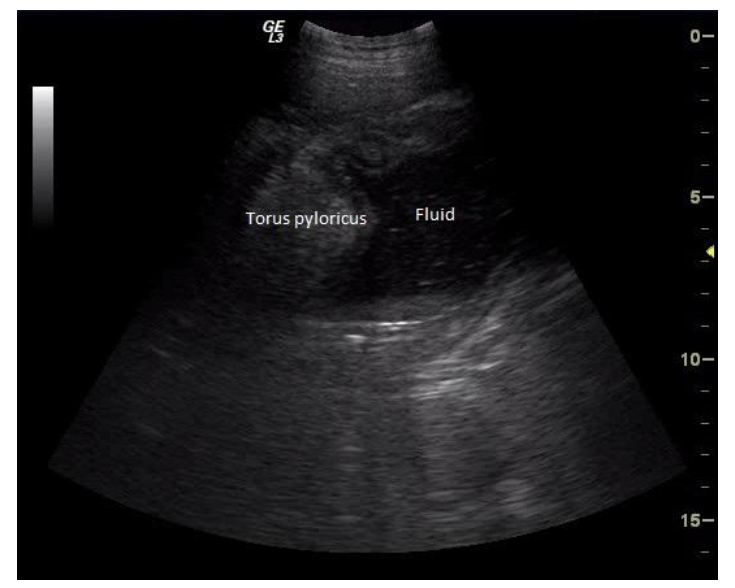

Fig.4 Part of small intestine (jejunum) trapped within tight fibrous band

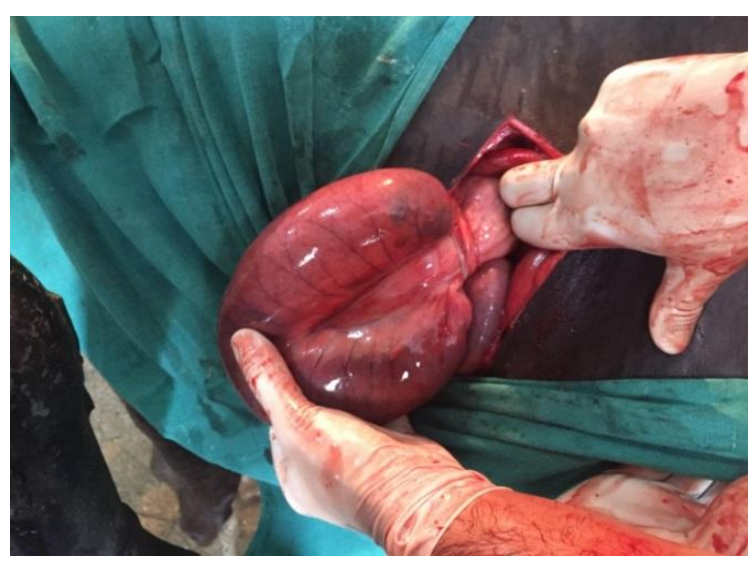

Fig.5 and 6 Histopathology of band like structure

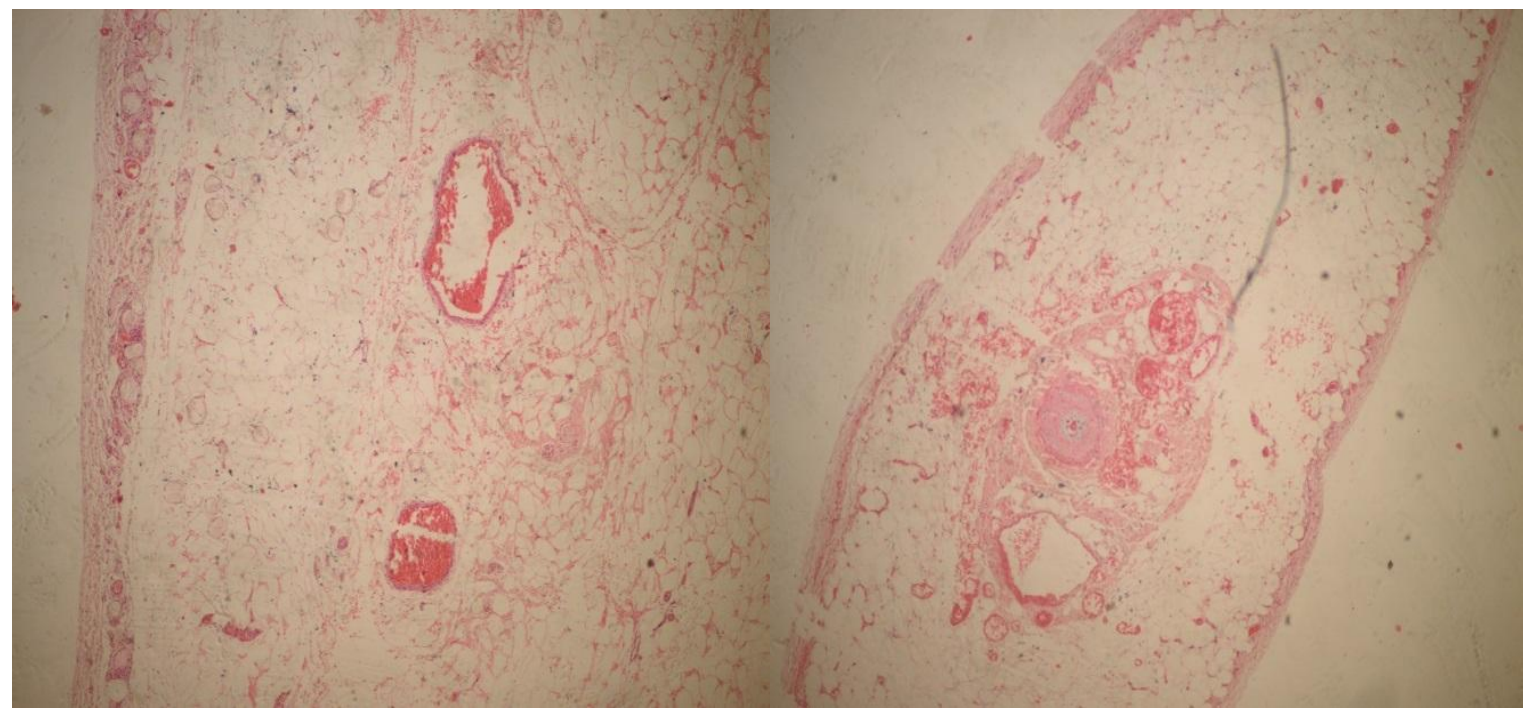


Exploratory laparotomyis indicated as a diagnostic and therapeutic aid in bovine animals suspected or diagnosed with ileus, as various tests including ultrasonographic findings are non-specific (Pardon et al., 2009) or in rare instances, ultrasonography in combination with simultaneous clinical examination, it may be useful to make a definitive diagnosis (Kumar et al., 2015).

In the present study fibrous band was found incarcerating the small intestine, the origin which could not be confirmed. Also, the cow had no history of a recent trauma, such as a fall, or recent calving (Matsui and Nukata, 2003); however, intestinal herniation may occur long after a tear has formed. Braun et al., (1989) reported caecal dilation and torsion and partial mesenteric torsion in Brown Swiss cattle that could lead to tension and tears of the greater omentum consequently entrapment of bowel.

Iatrogenic tears from previous surgeries have been reported in medical science (Blachar and Federle, 2002) and this cannot be ruled out in cattle as well (Pardon et al., 2009). Cited literature reports possible causes of omental or mesenteric rents through which hernias occur include trauma (Pardon et al., 2009) and congenital defects in the mesentery (Koller et al., 2001).

In conclusions, this case report puts on record the diagnosis and successful surgical management of a rare case of gut-tie due to fibrous band in a crossbred cow.

\section{Acknowledgements}

Authors are highly thankful to the Indian Council of Agricultural Research, New Delhi for providing financial support under 'All India Network Program on Diagnostic Imaging and Management of Surgical Conditions in Animals'

\section{References}

Anderson, D.E., P.D. Constable, St Jean, G and Hull, B.L. 1993. Small intestinal volvulus in cattle: 35 cases (19671992). Journal of the American Veterinary Medical Association. 203 (8): 1178-1183.

Baxter, G.M., B.J. Darien and Wallace, C.E. 1987. Persistent urachal remnant causing intestinal strangulation in a cow. Journal of the American Veterinary Medical Association. 191, 555-558.

Blachar, A., and Federle, M.P. 2002. Internal hernia: An increasingly common cause of small bowel obstruction. Seminars in Ultrasound, CT, and MRI. 23, 174-183.

Braun, U., Steiner, A and Bearth, G. 1989. Therapy and clinical progress of cattle with dilatation and torsion of the caecum. Veterinary Record. 125, 430433.

Coetzee, J.F., 2000. Partial obstruction of the small intestine in a cow. Veterinary Record. 147, 719-721.

Deprez, P., M. Hoogewijs, L. Vlaminck, K. Vanschandevijl, L. Lefere and Van Loon, G. 2006. Incarceration of the small intestine in the epiploic foramen of three calves. Veterinary Record. 158, 869-870.

Koller, U., C. Lischer, H. Geyer, C. Dressel and Braun, U. 2001. Strangulation of the duodenum by the uterus during late pregnancy in two cows. The Veterinary Journal. 162, 33-37.

Kumar Anil., A. Kumar, J. Mohindroo, T. Singh and Gupta, A. 2015. Diagnosis and surgical management of intussusception in cattle. Indian Journal of Veterinary Surgery. 36 (2): 116-118.

Lores, M., J.A. Haruna and Ortenburger, A. 2006. Bilateral 'gut-tie' in a recently castrated steer. Canadian Veterinary Journal. 47, 155-157. 
Matsui, T., and Nukata, S. 2003. Omental hernia immediately before the first parturition in a dairy cow. Journal of Livestock Medicine. 486, 761-765.

Mesaric, M., and Modic, T. 2003. Strangulation of the small intestine in a cow by a persistent urachal remnant. Veterinary Record.153, 688-689.

Pardon, B., G. Vertenten, I. Durie, J. Declercq, D. Everaert, P. Simoens and Deprez, P. 2009. Four cases of omental herniation in cattle. Veterinary Record. $165,718-721$.

Pearson, H., 1973. The treatment of surgical disorders of the bovine abdomen. Veterinary Record. 92, 245-254.
Robertson, J.T., 1979. Differential diagnosis and surgical management of intestinal obstruction in cattle. Veterinary Clinics of North America: Large Animal Practice. 1, 377-394.

Scott, P.R., A.E. Kinder, N.A. Blake and Hawe, C. 1997. 'Gut-tie' in a recently castrated steer. Veterinary Record. 140, 559-560.

Singh, G., N.S. Saini, A. Kumar, S.K. Mahajan, J. Mohindroo and Sood, N.K. 2017. Diagnosis and outcome of surgical management of intestinal obstruction in bovine. Indian Veterinary Journal. 94(1), 32-35.

\section{How to cite this article:}

Harmanpreet Singh Sodhi, Balreet Singh Sidhu and Ashwani Kumar. 2019. Surgical Management of Gut-Tie in a Cow. Int.J.Curr.Microbiol.App.Sci. 8(08): 542-547.

doi: https://doi.org/10.20546/ijcmas.2019.808.064 